\title{
Maternal High-Fat Diet Programs Renal Peroxisomes and Activates NLRP3 Inflammasome-Mediated Pyroptosis in the Rat Fetus
}

\section{Pei Zhou* \\ Hongbo Guan* \\ Yanyan Guo \\ Liangliang Zhu \\ Xiaomei Liu (D)}

Key Laboratory of Maternal-Fetal Medicine of Liaoning Province,

Department of Obstetrics and Gynecology, Shengjing Hospital of China Medical University, Shenyang, I I 0004

People's Republic of China

*These authors contributed equally to this work
Correspondence: Xiaomei Liu Key Laboratory of Maternal-Fetal Medicine of Liaoning Province, Department of Obstetrics and Gynecology, Shengjing Hospital of China Medical University Shenyang, II0004,

People's Republic of China

Tel +86-18940255055

Email liuxm@cmu.edu.cn
Purpose: Maternal obesity impairs kidney development and function of the offspring and leads to a greater risk of kidney disease in adulthood. The present study aimed to investigate the link between peroxisomes, oxidative stress (OS), and inflammasomes in the fetal kidney of maternal obesity rats and to explore the potential therapeutic effects of the antioxidant pyrroloquinoline quinone (PQQ).

Methods: Maternal obesity rats were developed by administration of a high fat diet plus supplementation with PQQ (40 mg/kg body weight) as a potential therapy. Renal histology was observed by Periodic Acid-Schiff staining. The expression profiles of peroxins, fatty acid $\beta$-oxidation enzymes, antioxidants, and the regulators of the unfolded protein response (UPR) pathway and NLRP3 inflammasome were analyzed in the kidneys and tubular epithelial cells (TECs) from near-term fetuses (embryonic day 20).

Results: The present work revealed that: 1) a maternal high fat diet (MHF) led to higher blood pressure in adult offspring; 2) MHF led to downregulation of peroxisome markers PEX3 and 14 in fetal kidneys; 3) the antioxidant SOD2 and catalase were decreased, and oxidative stress marker Ephx2 was increased; 4) MHF-induced activation of the UPR pathway; 5) the KEAP1-NRF2 pathway was activated; 6) activation of the NLRP3 inflammasome led to secretion of pro-inflammation factors; 7) in TECs, the changes in PEXs and NLRP3 are similar to tissues, but UPR and NRF2 pathways showed opposite trends; 8) and the antioxidant PQQ alleviated maternal lipotoxicity by decreasing ROS levels and inhibiting activation of ER stress and inflammasome in fetal kidney.

Conclusion: A maternal high fat diet decreased the number of peroxisomes, subsequently activated OS and inflammasomes, resulting in pyroptosis and apoptosis in fetal kidney. The antioxidant PQQ served a protective role against the effects of lipotoxicity on kidney programming and, thus, is a potential candidate to prevent maternal obesity-induced renal programming.

Keywords: maternal obesity, kidney, peroxisome, oxidative stress, inflammasome

\section{Introduction}

Obesity has become an important public health concern worldwide due to the substantially increased prevalence over the past few decades. ${ }^{1}$ Epidemiological studies have reported that about $20-30 \%$ of pregnant women are overweight or obese, ${ }^{2}$ and, thus, are at a greater risk of developing complications of pregnancy, such as gestational diabetes, hypertension, and preeclampsia. ${ }^{3}$ The offspring of women who are obese during pregnancy are exposed to a milieu of adverse 
intrauterine events during key periods of embryonic development and are at greater risks of metabolic syndrome, which has been linked to the development of hypertension, obesity, and type 2 diabetes. $^{4-6}$ Evidence from human studies indicates that maternal obesity adversely affects kidney programming, thereby contributing to a greater risk of chronic kidney disease (CKD) in adulthood. ${ }^{7}$ Animal studies have also indicated that a lipotoxic intrauterine environment adversely impacts kidney development and function. ${ }^{8}$ Kidney programming greatly promotes susceptibility to $\mathrm{CKD},{ }^{9}$ thus further understanding of the mechanisms underlying kidney development in the fetuses of women who are obese during pregnancy is highly warranted to prevent CKD.

Maternal obesity impairs normal embryonic development through deregulation of metabolic homeostasis, the accumulation of reactive oxygen species (ROS) and reactive nitrogen species (RNS), inflammatory responses, and consequent cell death. ${ }^{10-12}$ ROS/RNS are free radicals, which include hydrogen peroxide $\left(\mathrm{H}_{2} \mathrm{O}_{2}\right)$, superoxide anion $\left(\mathrm{O}_{2} \cdot-\right)$, hydroxyl radical $(\mathrm{OH} \cdot)$, and nitric oxide (NO). ${ }^{13}$ Oxidative stress (OS) originates from an imbalance between the generation of ROS and RNS and associated detoxification mechanisms. ${ }^{14}$ The peroxisome is a ubiquitous organelle in eukaryotic cells that plays important roles in the production and degradation of cellular ROS/RNS. Peroxins (PEXs) regulate the biogenesis and degradation of peroxisomes. Thus, mutations of PEX genes can cause defects in peroxisome proliferation and function. ${ }^{15}$ Moreover, OS is bidirectionally and synergically related to inflammation, as OS induces inflammation and vice versa, while ROS have been identified as activators of the NLRP3 (NOD-, LRR- and pyrin domaincontaining protein 3) inflammasome ${ }^{16}$ ROS is also an inducer of ER stress, excessive ROS production leads to ER stress-induced cell death. ${ }^{17}$ Furthermore, in response to OS, the transcription factor NF-E2 p45-related factor 2 (NRF2) induces the expression of antioxidant genes, including $\mathrm{NAD}(\mathrm{P}) \mathrm{H}$ quinone oxidoreductase 1 (NQO1), heme oxygenase-1 (HO-1), and glutamate cysteine ligase catalytic subunit (GCLC), thus playing a vital role in protection from toxicity and stress-induced inflammation. Activation of NRF2 inhibits ROS-NLRP3 signaling and attenuates OS. ${ }^{18}$ However, little is known about the programming effect of obese pregnancy on the link among peroxisomes, the NRF2 pathway, and inflammasomes during critical periods of kidney development.
Therefore, female rats fed a high fat diet were used to investigate whether maternal obesity impaired the production and function of peroxisomes and inflammasomes in the fetal kidney. With the primary cultured tubular epithelial cells (TECs), we further investigate whether the activation of OS and inflammasome occurred in renal tubular epithelial cells and lead to renal tubular dysplasia. Also, the potential of pyrroloquinoline quinone (PQQ), a novel dietary antioxidant against harmful OS, ${ }^{19}$ was explored as a countermeasure to protect offspring against high fat toxicity during pregnancy and lactation.

\section{Materials and Methods}

\section{Animals}

The animal study protocol was approved by the Animal Research Committee of Shengjing Hospital of China Medical University (Shenyang, China; approval number, 2018PS288K). All experiments were performed in accordance with the guidelines of the National Institutes of Health. A rat model of maternal obesity was established with a high fat diet. In brief, Wistar rats (age, 5 weeks) were randomly assigned to one of three groups $(n=10)$, a control group that was fed a normal diet (10\% kcal from fat), a maternal high fat (MHF) group that was fed a high fat diet $(60 \% \mathrm{kcal}$ from fat) matched for micronutrients (Supplemental Table 1), or a HF-PQQ group that was fed a high fat diet and supplemented with PQQ (40 mg/kg body weight, dissolved in water) by gavage through gestation and lactation. The dose we choose is based on previous reports on PQQ efficacy and toxicity research in rats. $^{20}$ The diets were fed to female rats ad libitum for 5 weeks before mating and throughout pregnancy and lactation. At $\sim 10$ weeks of age, the females were mated with males fed a normal diet and considered as pregnant by the presence of a vaginal plug. Mother rats were individually housed.

Some pups were delivered by caesarean section on embryonic day 20 (E20) to ensure that the near-term fetuses from both groups are of the same gestational ages. A portion of the right kidney was fixed in $4 \%$ paraformaldehyde and the other kidneys were stored at $-80^{\circ} \mathrm{C}$ until analysis. Other pups were delivered spontaneously, nourished by the mother, and fed a normal diet after weaning. At 12 weeks, in the awake and quiet state, systolic blood pressure (SBP) was recorded using tail cuff with a sphygmomanometer (BP-98A, Softron), and each rat was measured 4 times to get the average values. 
A metabolic cage was used to obtain 12-h urine samples for measurement of renal functional parameters (CREA, protein, UA etc.) with Architect ci16200 chemistry analyzer (Abbott Laboratories, Abbott Park, IL, USA). The offspring of at least three separate litters were used for analyses. The data presented herein were obtained from males (except for E20 fetuses) to exclude any potential bias caused by sex-specific hormone levels.

\section{Renal Histology}

Paraformaldehyde (4\%)-fixed and paraffin-embedded kidney tissue sections $(3 \mu \mathrm{m})$ were subjected to Periodic Acid-Schiff (PAS) staining and nephron was counted under $200 \times$ magnification in five areas of three sections from six fetuses per group. Immunohistochemistry (IHC), and immunofluorescence (IF) staining were conducted in accordance with standard protocols using commercial assay kits and antibodies (Supplemental Table 2). In brief, sections were dewaxed, antigen-repaired, blocked for endogenous peroxidase and nonspecific antigens, then incubated with primary antibodies at $4^{\circ} \mathrm{C}$ overnight. As a negative control, the primary antibody was omitted with no staining above background as a result (Supplement Figure 1). Finally, images of the sections were captured with an ECLIPSE Ti fluorescence microscope (Nikon | Corporation, Tokyo, Japan) or a laser scanning confocal microscope. Quantitative analysis of fluorescence intensity was conducted in three areas of three sections from six fetuses per group with Image J software (https://imagej. nih.gov/ij/).

\section{Cell Culture}

Renal tubular epithelial cells (TEC) were isolated from control and MHF fetal kidneys. Briefly, fetal kidneys were decapsulated and minced in cold PBS, then the cortex was retained and digested in DMEM-F12 medium (Biological Industries) with Collagenase IV (1 mg/mL, Solarbio) for 30 minutes at $37{ }^{\circ} \mathrm{C}$. Afterwards, Collagenase IV activity was neutralized with growth medium [DMEM-F12 medium and 10\% FBS (HAKATA) containing 100 unit $/ \mathrm{mL}$ penicillin and $0.1 \mathrm{mg} / \mathrm{mL}$ streptomycin]. The cell suspension was sieved through a nylon cell strainer $(\varnothing 100 \mu \mathrm{m})$. The filtrate was centrifuged and the pellet was resuspended in growth medium before plating onto culture dishes. Cells were cultured at $37^{\circ} \mathrm{C}$ in a $\mathrm{CO} 2$ incubator with medium changes every 2 days until collected. The cells used in this study were passage 2. IF staining of CK18, a specific marker of epithelial cell, confirmed the cells were from tubular epithelial (Supplement Figure 2).

\section{Determination of Malondialdehyde (MDA) Levels}

MDA level in the fetal kidney was measured with a CheKine ${ }^{\mathrm{TM}}$ Lipid Peroxidation (MDA) Assay Kit (ktb1050; Abbkine, Wuhan, China) and are presented as nmol MDA/mg protein.

\section{Quantitative PCR}

Total RNA was extracted with RNAiso reagent (TaKaRa Biotechnology Co., Ltd., Dalian, China), assayed for quantity and purity, then reverse-transcribed into complementary DNA (cDNA) with a cDNA synthesis kit (RR047A; TaKaRa Biotechnology Co., Ltd.). The cDNA was amplified using a 7500 fast real-time PCR system (Applied Biosystems, Carlsbad, CA, USA) with the primers listed in Supplementary Table 3. Data were calculated with the $2^{-\Delta \Delta \mathrm{Ct}}$ method after normalization against $\beta$-actin as an internal control.

\section{Western Blot Analysis}

Total protein was extracted with SDT buffer $(4 \%[\mathrm{w} / \mathrm{v}]$ sodium dodecyl sulfate, $0.1 \mathrm{M}$ Tris $/ \mathrm{HCl} \mathrm{pH} 7.6,0.1$ $\mathrm{M}$ dithiothreitol) and quantified with a bicinchoninic acid protein assay kit. Equal amounts of protein $(20 \mu \mathrm{g})$ were separated by sodium dodecyl sulphate-polyacrylamide gel electrophoresis and transferred onto a polyvinylidene difluoride membrane, which was cut into several strips that were blocked and incubated with primary antibodies (Supplementary Table 2). After probing with secondary antibodies, the blots were visualized with Enhanced Chemiluminescence Plus reagent (EMD Millipore Corporation, Billerica, MA, USA).

\section{Statistical Analysis}

The results are presented as the mean \pm standard error of the mean. The Student's two-tailed $t$-test was used for comparisons between two groups. Multigroup comparisons of the means were carried out by one-way analysis of variance (ANOVA) test with Dunnett's T3 test or Fisher's LSD test as the post hoc test. A probability $(p)$ value of $<0.05$ was considered statistically significant. 


\section{Results}

\section{Animal Phenotype}

There was no significant difference in the litter size and resorption rate between the MHF and control groups (data not shown). The birth weight of the neonates was slightly lower in the MHF group than the control group. The kidney weight and kidney index were higher in the nearterm fetuses of the MHF group (E20), but there was no significant difference at 12 weeks between two groups. As compared with the control group, SBP was significantly increased at 12 weeks in the MHF group (Figure 1A). Serum UA and urine protein were increased. Serum
CREA was not varied, urine CREA was mild increased thus ratio of blood/urine was moderately decreased, though this did not reach significance. These indices indicated mild renal injury in MHF adult offspring (Supplemental Table 4).

\section{Kidney Histopathology Development Indices}

PAS staining showed that MHF had little effect on the number of nephrons in the fetal kidney (Figure 1B). $\mathrm{AQP1}$, the marker of proximal tubular epithelium was reduced in MHF fetal kidney (Figure 1C). Immunostaining
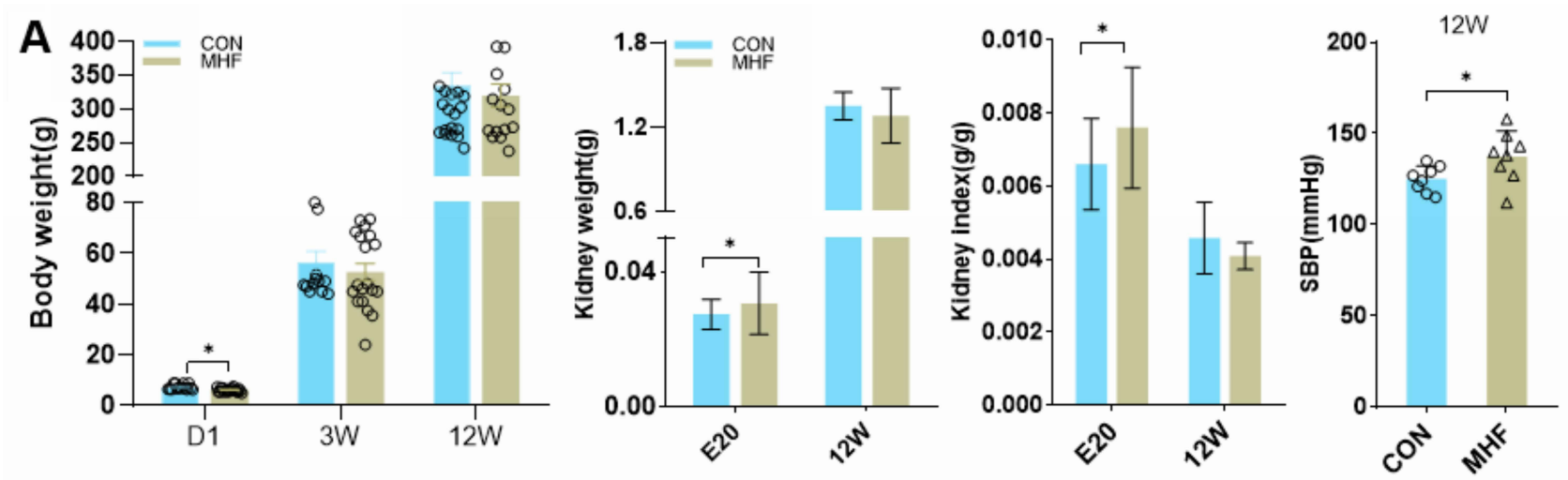

B
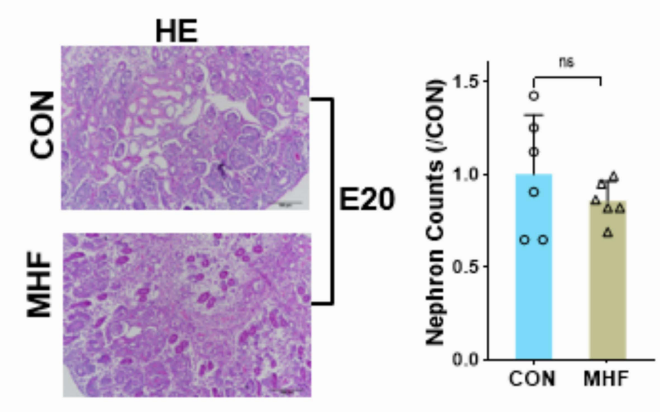

C
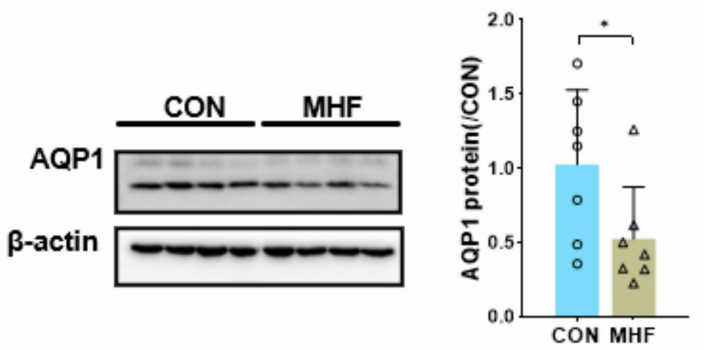

D
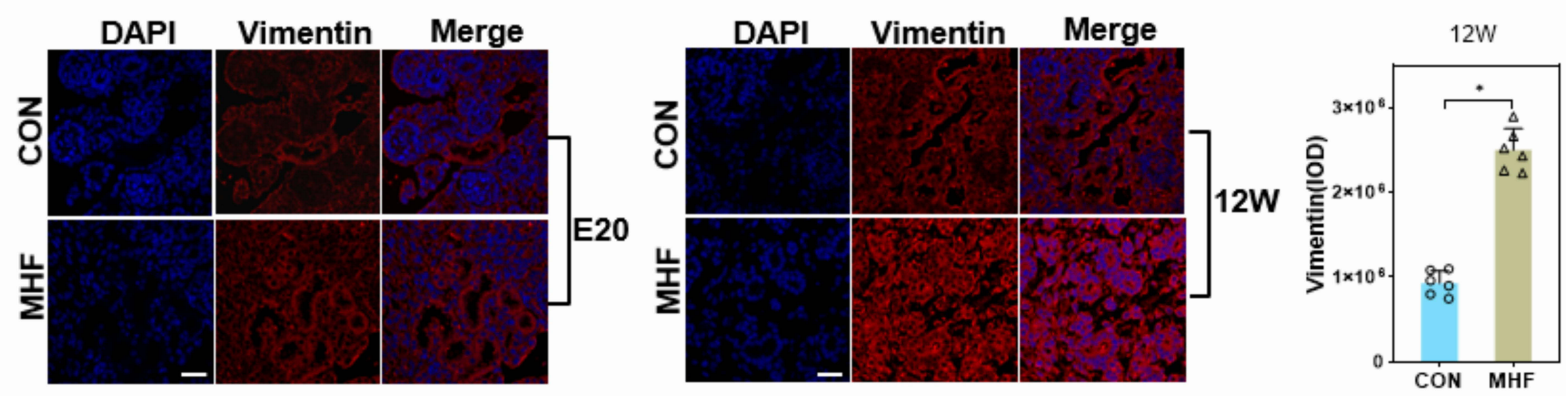

Figure I Effect of a high fat diet of pregnant rats on kidney growth of offspring. (A) Body weight, kidney weight, kidney index, and systolic blood pressure (SBP) at the indicated time points in both groups. (B) Representative PAS-stained kidney sections and a graph of nephron counts. Images were captured at a magnification of 200x; scale bar $=100 \mu \mathrm{m}$. ( $\mathrm{n}=6$ per group, ${ }^{*} p<0.05$ vs control). (C) Western blot analysis of AQPI proteins ( $\mathrm{n}=7$ per group, ${ }^{*} p<0.05$ vs control). (D) Immunofluorescence (IF) staining of vimentin in both groups at E20 or $12 \mathrm{~W}$ (magnification of $800 \times$; scale bar $=50 \mu \mathrm{m}$ ). 
against vimentin showed tubular fibrosis in the adult offspring of the MHF group, but not the fetuses (Figure 1D).

\section{Effect of MHF on PEXs in Fetal Kidney}

As is shown in Figure $2 \mathrm{~A}-\mathrm{C}$, the protein expression levels of PEX3, 14, and 19 were significantly downregulated in the fetal kidney of the MHF group, while PEX5 was upregulated. The qPCR analysis showed that the mRNA levels of PEX1, 7, and 26 were increased, while those of PEX3 and 14 were decreased, consistent with the protein data. There was no significant difference in the transcript levels of PEX11A, $11 \mathrm{~B}$ and 16 between the two groups. Catalase (CAT), which degrades $\mathrm{H}_{2} \mathrm{O}_{2}$ and is an established marker of peroxisomes, was significantly decreased in the fetuses of the MHF group, as determined by IF co-staining with PEX14 (Figure 2C).

\section{Regulators of Fatty Acid $\beta$-Oxidation (FAO) in Fetal Kidney}

FAO in the peroxisome generates ROS or RNS as byproducts. Peroxisomal membrane protein (PMP) 70, which is involved in PMP sorting and the transport of fatty acids, was also downregulated in the MHF group, as determined by Western blot, q-PCR and IF. The protein levels of hydroxysteroid 17-beta dehydrogenase 4 (HSD17b4) and enoyl-CoA hydratase and 3-hydroxyacyl CoA dehydrogenase (EHHADH), which are rate-limiting enzymes that catalyze the second step of FAO, were upregulated and downregulated, respectively. However, mRNA levels of ACOX1 and 2, the rate-limited enzymes that catalyze the first step of FAO, were unaffected by MHF (Figure 3A-C).

\section{Antioxidant System in Fetal Kidney}

The endogenous antioxidants superoxide dismutase 2 (SOD2), and CAT were significantly downregulated in the MHF group. In contrast, mRNA level of epoxide hydrolase 2 (EPHX2), an antioxidant mainly located in the peroxisomes and cytosol, was increased. There was no significant difference in the mRNA levels of the antioxidants peroxiredoxin 5 (PRDX5) and glutathione S-transferase kappa 1 between the MHF and control groups. Interestingly, NOS2 showed a decrease in mRNA level, but an increase in protein expression, evidenced by IF staining (Figure 4A-C). Augmented levels of MDA, an end-product of lipid peroxidation, reflect OS in the fetuses of the MHF group (Figure 4D). Further, protein interaction analysis of the detected differentially expressed genes between two groups was conducted using the STRING database
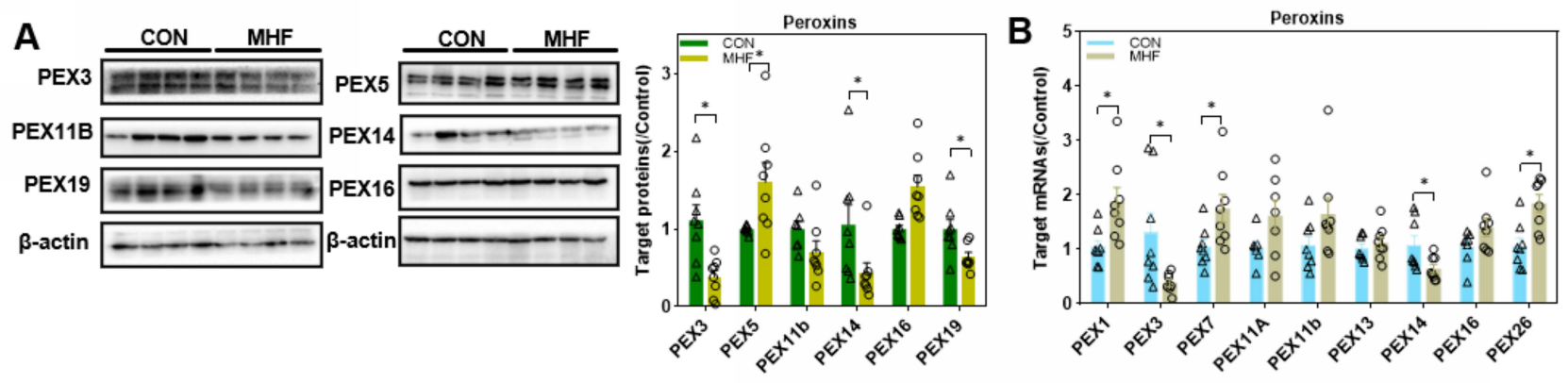

C
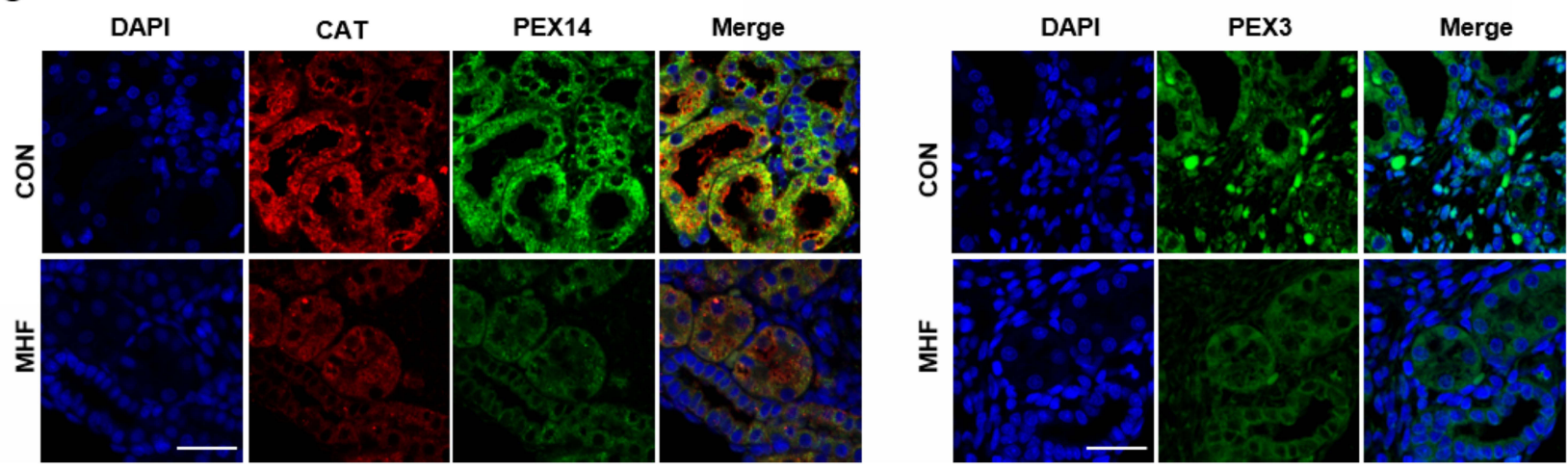

Figure 2 Maternal high fat (MHF) diet induced dysregulation of peroxins (PEXs) in the fetal kidney. (A) Western blot analysis of PEXs proteins. (B) qPCR analysis of the PEXs mRNA levels. ( $n=7-8$ per group, ${ }^{*} p<0.05$ vs control). (C) IF staining of PEXI4 and catalase colocalization and distribution of PEX 3 in the fetal kidney (magnification of $800 \times$; scale bar $=50 \mu \mathrm{m})$. 
A

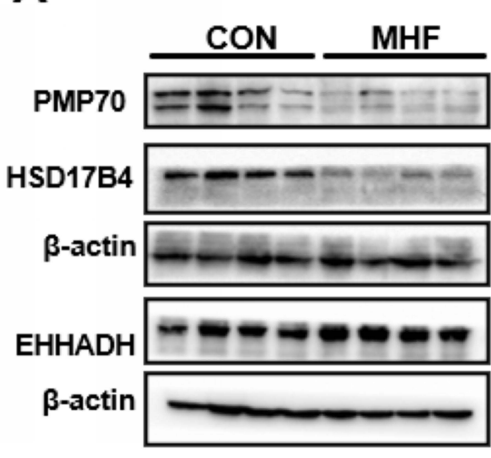

C
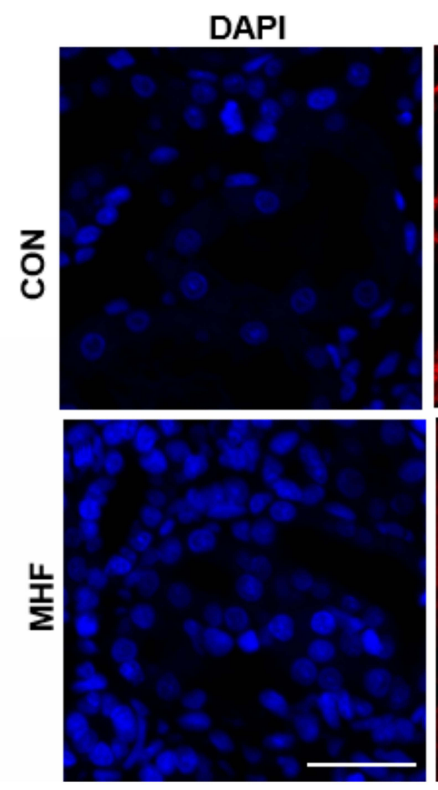

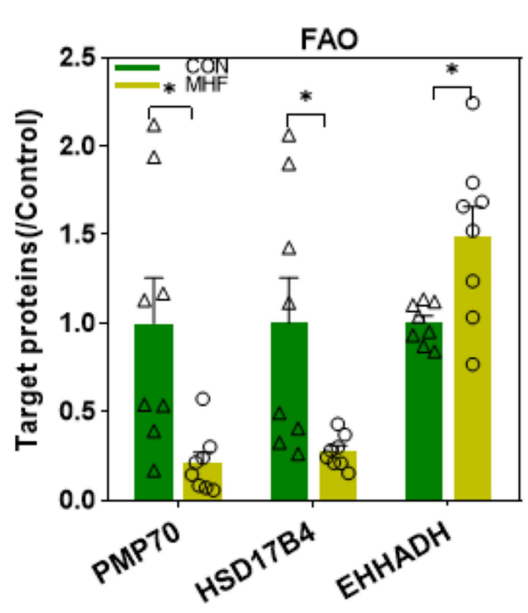

B
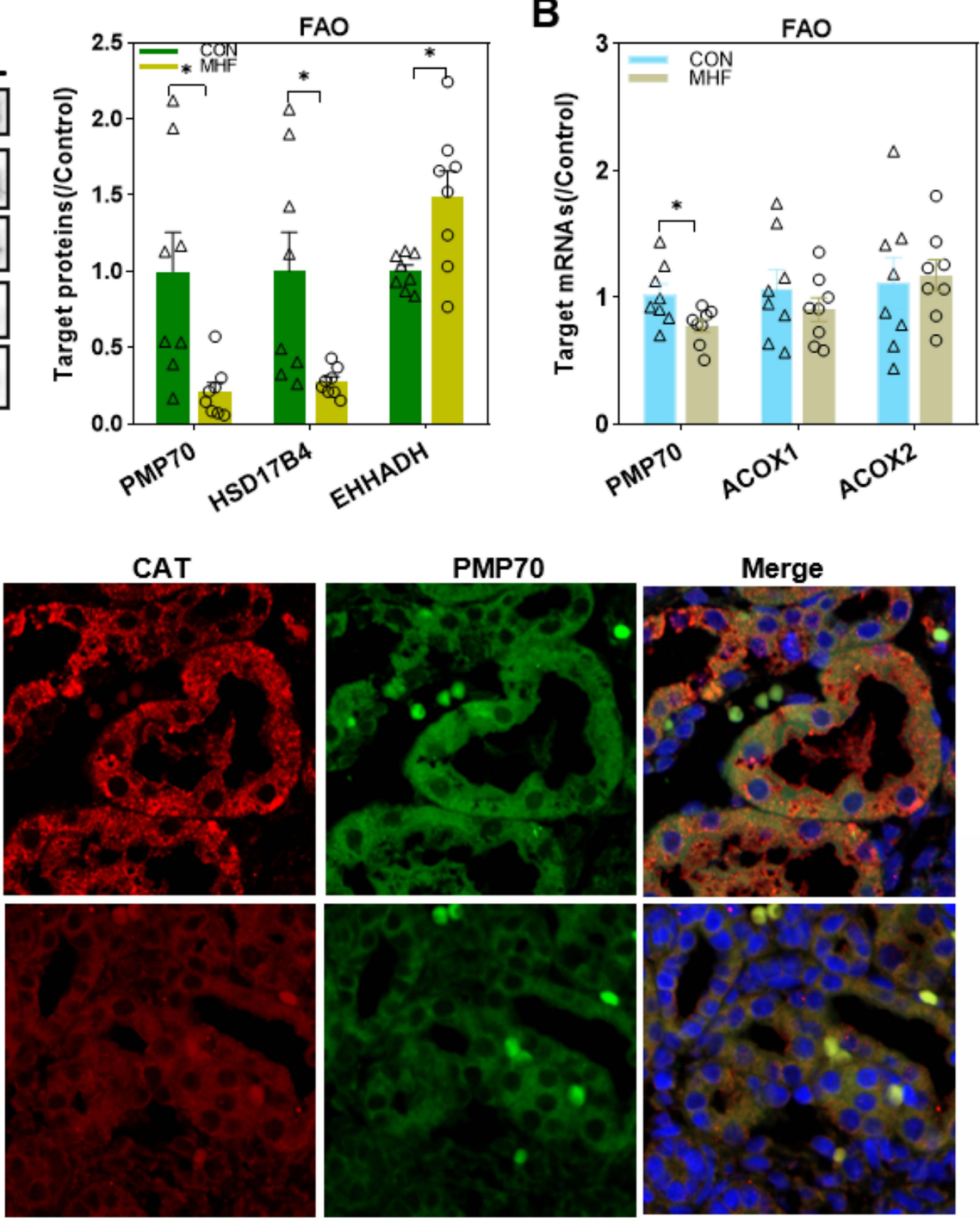

Figure 3 Effects of maternal high-fat on fatty acid $\beta$-oxidation (FAO) regulators in the fetal kidney. (A) Western blot analysis of FAO regulators. (B) The qPCR analysis of the mRNA levels of FAO regulators. ( $n=8$ per group, ${ }^{*} p<0.05$ vs control). (C) IF staining of Peroxisomal membrane protein (PMP) 70 and catalase (CAT) co-localization in the fetal kidney (magnification of $800 \times$; scale bar $=50 \mu \mathrm{m}$ ).

(https://string-db.org/), to show that all the differentially expressed antioxidants and FAO regulators interrelated with peroxins and PEX3 /14 seemed to be the hub genes. (Figure 5A). Kyoto Encyclopedia of Genes and Genomes (KEGG) pathway analyses (http://www.genome.jp/kegg) showed that each cluster of PEXs function synergistically in peroxisome biogenesis have the same change trend (Figure 5B).

\section{Activation of the NRF2 Pathway in Fetal Kidney}

Western blot analyses showed decreased expression of KEAP1 and enhanced expression and nuclear translocation of NRF2 in the fetal kidney of the MHF group. The qPCR analyses showed that the expression levels of NRF2 and associated downstream genes (ie, NQO1, HO-1, and glutathione reductase) tended to be enhanced, although the latter two did not reach statistical significance due to high individual variations (Figure 6A and $\mathrm{B}$ ).

\section{Activation of Inflammasomes and Pyroptosis in Fetal Kidney}

The qPCR, Western blot and IF analyses showed enhanced expression of thioredoxin interacting protein (TXNIP), purinergic receptor $\mathrm{P} 2 \mathrm{X} 7$, and indicators of the inflammasome and pyroptosis (NLRP3, apoptosis-associated speck-like protein containing a CARD [ASC], and interleukin [IL]-18) in fetuses of the MHF group, indicating activation of inflammasomes. 


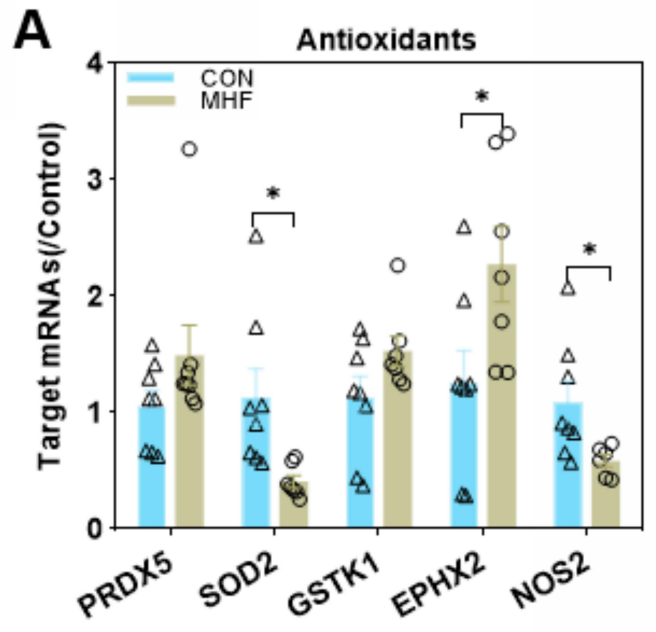

C
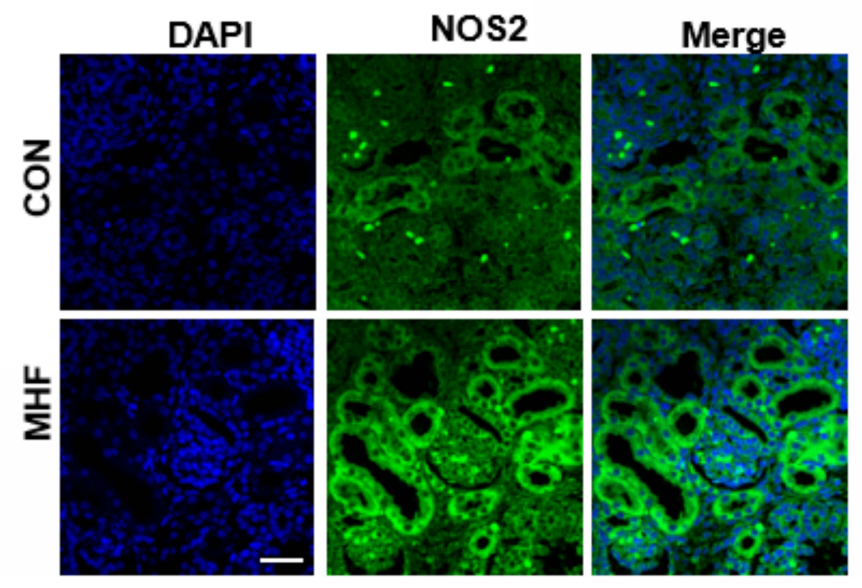
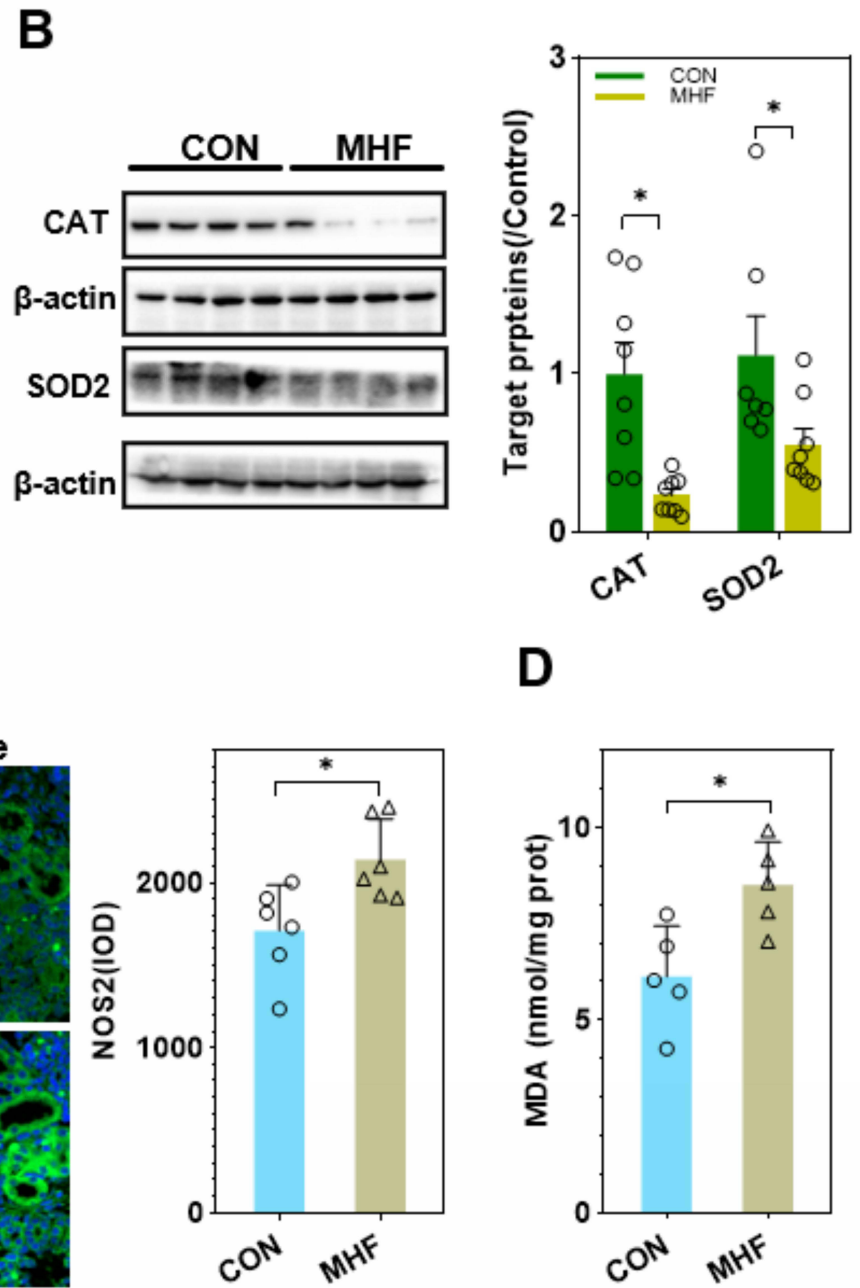

D

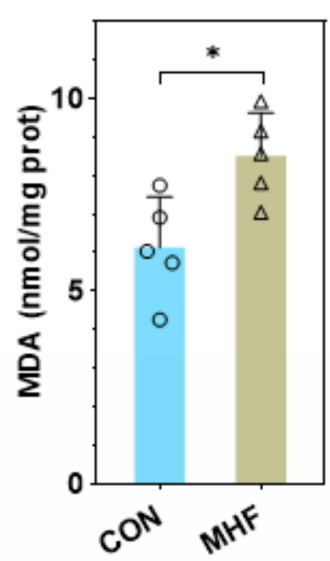

Figure 4 Effects of MHF on antioxidants in the fetal kidney. (A) The qPCR analysis of the mRNA level of antioxidants in both groups. (B) Protein expression of the antioxidants CAT and superoxide dismutase (SOD)2. ( $n=7-8$ per group, $*_{p}<0.05$ vs control). (C) IF staining showing the locations and expression of nitric oxide synthase (NOS) 2 in the fetal kidney of CON and MHF group $(400 \times$, scale bar $=50 \mu \mathrm{m}, \mathrm{n}=6$ per group, $* p<0.05$ vs control). (D) MDA levels in the fetal kidney of both groups. ( $\mathrm{n}=5$ per group, $*_{p}<0.05$ vs control).

Cleavage of IL-1 $\beta$, IL-18, and caspase-1 was increased, although the mRNA level of IL-1 $\beta$ was unchanged. Meanwhile, cluster of differentiation (CD)68 and the F4/80 antigen (Emr1) were increased in the fetuses of the MHF group, indicating that MHF induced the accumulation of macrophages and secretion of proinflammatory cytokines (Figure 7A-C).

\section{Activation of Endoplasmic Reticulum Stress (ERS) and Apoptosis in Fetal Kidney}

A previous report suggested that maternal obesityinduced ERS and subsequent maldevelopment of the hypothalamus. $^{21}$ A high fat diet-induced upregulation of the ERS regulators Activating transcription factor (ATF)4/6, PKR-like ER kinase (PERK), DNA damage- inducible transcript 3, also known as C/EBP homologous protein (CHOP), inositol-requiring enzyme 1 (IRE1), and spliced X-box binding protein $1(\mathrm{~s}-\mathrm{XBP} 1)$ in the fetal kidney, which indicated activation of ERS (Figure 8AC). eIF2a was unaffected by maternal high fat, however, the p-eIF2a was enhanced in MHF fetus. In addition, expression of the pro-apoptosis factor Bax was increased, while that of the anti-apoptosis factor Bcl-2 was decreased. The expression levels of cleaved caspase9 and caspase- 8 were increased, suggesting enhanced mitochondria-mediated apoptosis (Figure 8D and E).

\section{Effect of MHF on TECs in Fetus}

Q-PCR analysis revealed that most of the tested PEXs showed a trend for reduction in primary culture TECs with exposure to MHF, though only the expression differences of Pex1,3,5,7,16 

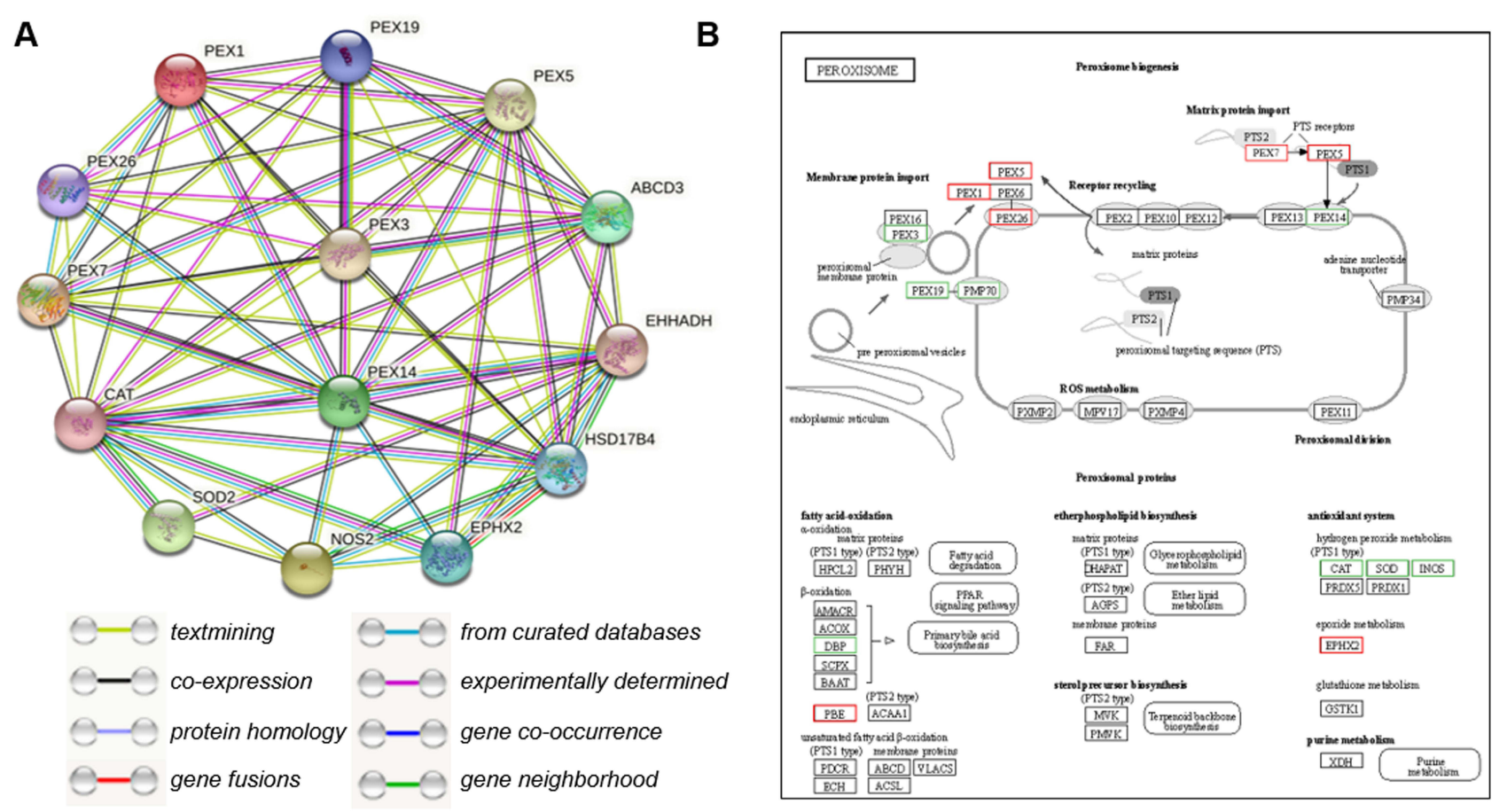

Figure 5 Protein interaction analysis. (A) STRING Protein-Protein Interaction network involving the detected differentially expressed genes. (B) KEGG pathway analysis of differentially expressed genes enriched in peroxisome-related pathways. Red indicates an increase in expression and green, a decrease.
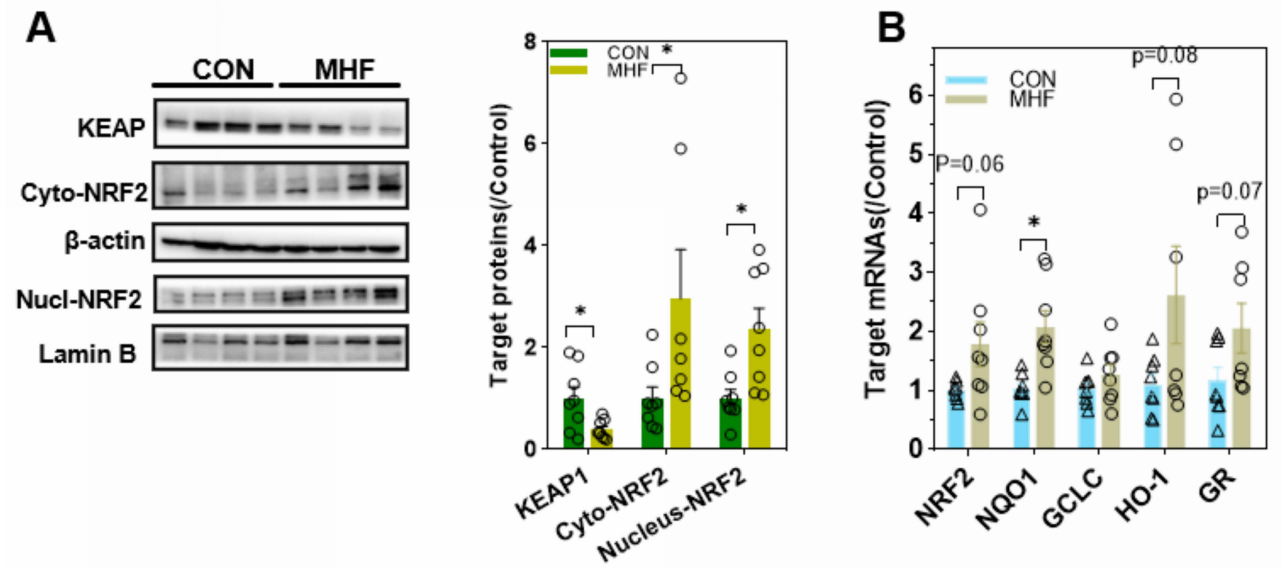

Figure 6 MHF triggered NRF2 activation in the fetal kidney. (A) Western blot analysis of the expression of Kelch-like ECH associated protein (KEAP)I and nuclear translocation of NF-E2- related factor 2 (NRF2). (B) qPCR analysis of the mRNA levels of NRF2 and associated downstream genes. * $p<0.05$ vs control; $(n=7-8$ per group, $*_{p}<0.05$ vs control).

were statistically significant. FAO rate-limiting enzymes Acox 1 and Hsd17b4 showed the tend to decrease though did not reach statistical significance, while Acox2 was enhanced in mRNA level. The mRNA levels of endogenous antioxidants CAT, Ephx2 and Prdx 5 were decreased. In contrast, Sod2 was enhanced, contrast to the result of renal tissues. Unexpectedly, NRF2 downstream genes and ERS regulators were all significantly decreased in mRNA level. Inflammasome genes NLRP3 and ASC were increased in mRNA level (Figure 9).

\section{Effect of PQQ on Fetal Development}

PQQ exposure increased fetal weight, but not fetal kidney weight, thereby decreasing the kidney index as compared with MHF offspring. PQQ also ameliorated MDA levels (Figure 10A) and the abnormal renal function indices, including urine proteins and uric acid to various degrees (Supplemental Table 4).

The qPCR results showed that PPQ treatment restored the mRNA levels of PEX3, PMP70, and CAT, consistent 

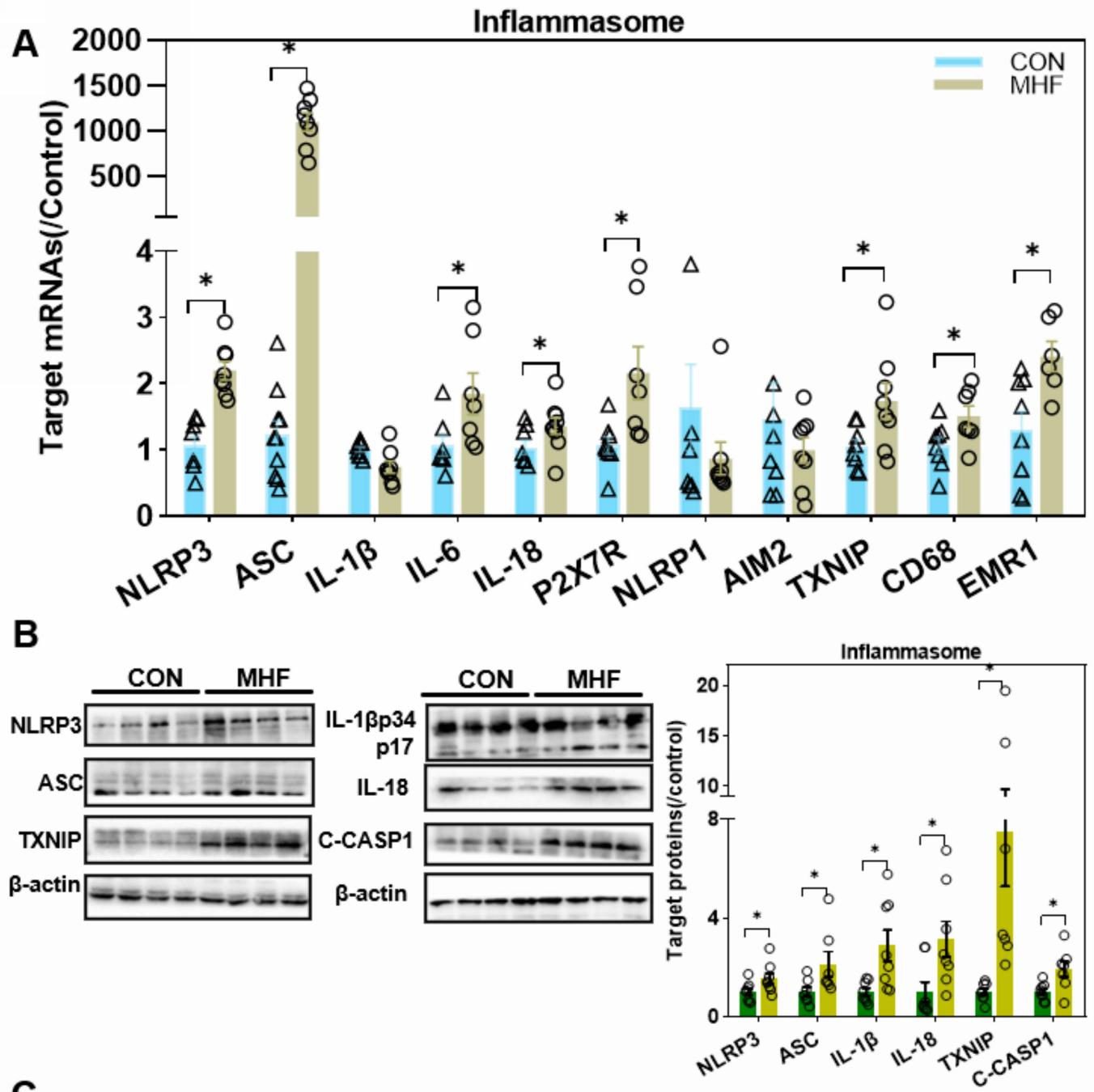

C
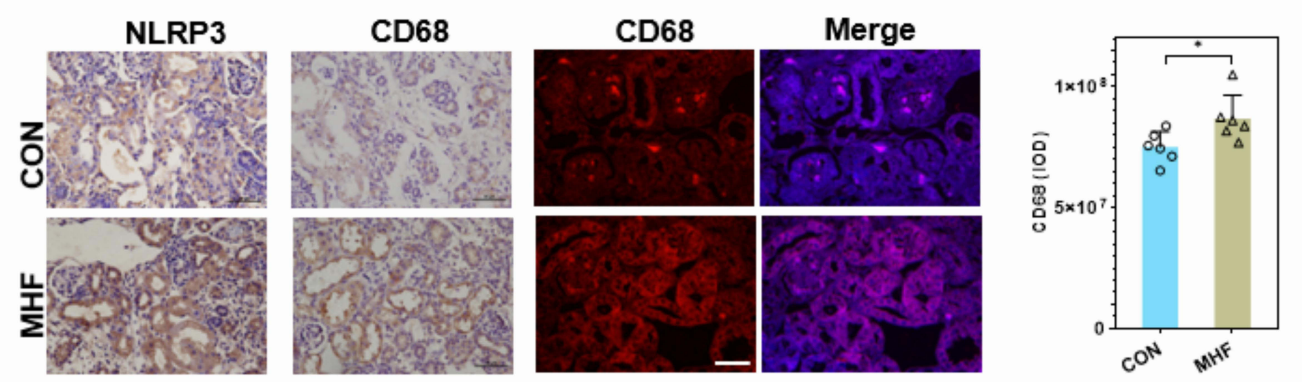

Figure 7 MHF induced activation of the NLR family, pyrin domain-containing (NLRP)3 inflammasome in the fetal kidney. (A) The qPCR analysis of mRNA levels of genes associated with the inflammasome in the fetal kidney. (B) Expression levels of protein associated with the NLRP3 inflammasome in the fetal kidney. $\left(n=7-8\right.$ per group, ${ }^{*} p<$ 0.05 vs control). (C) IHC of NLRP3 and CD68 and IF staining of CD68 showed the expression and location of NLRP3, and CD68 in the fetal kidney (magnification of 400x; scale bar $=50 \mu \mathrm{m})$.

with expectations. Instead, the mRNA levels of PEX14, SOD2 were further diminished. The mRNA expression of PEX1, 7, and 26 was unaffected by PQQ treatment. Q-PCR and IF staining showed that NOS2 expression was decreased which indicated that PQQ reversed the activation of NOS2 induced by MHF. PQQ also partly alleviated the abnormal activation of the NRF2 pathway and inflammasomes by diminishing the mRNA levels of NRF2 target genes (GCLC, NQO1) and inflammasome-related genes (TXNIP and ASC). Compared with MHF group, PQQ 

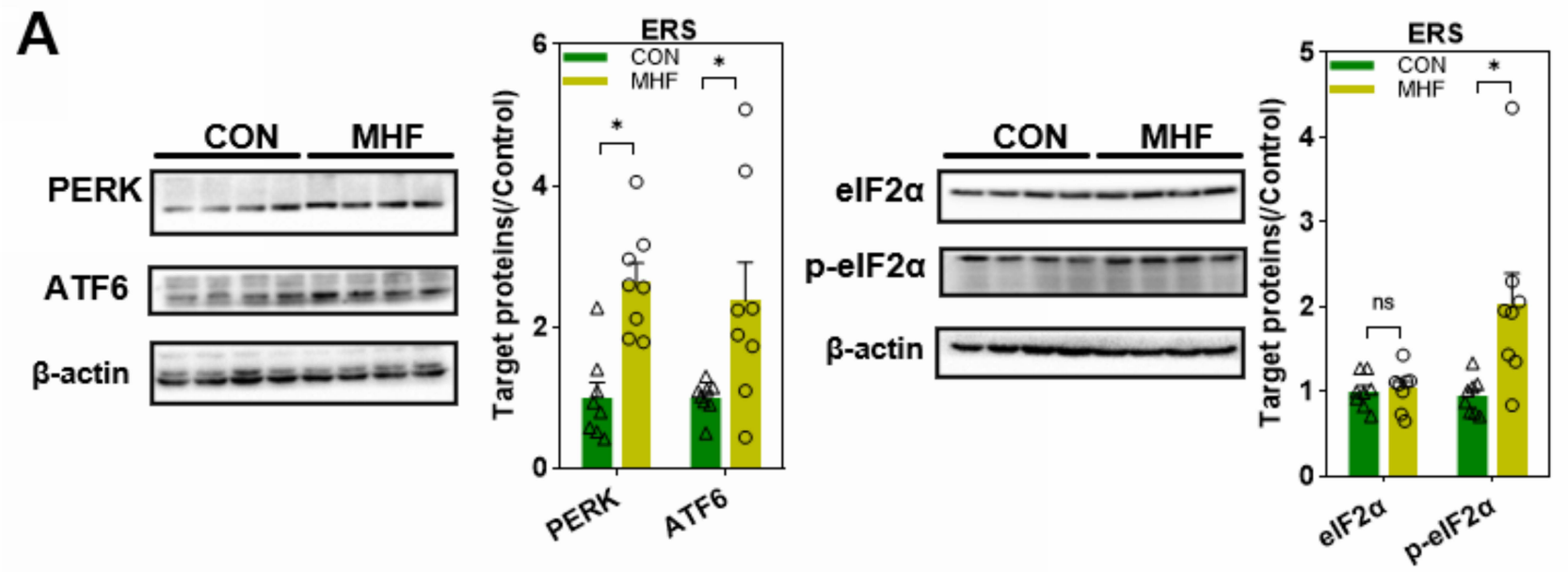

B
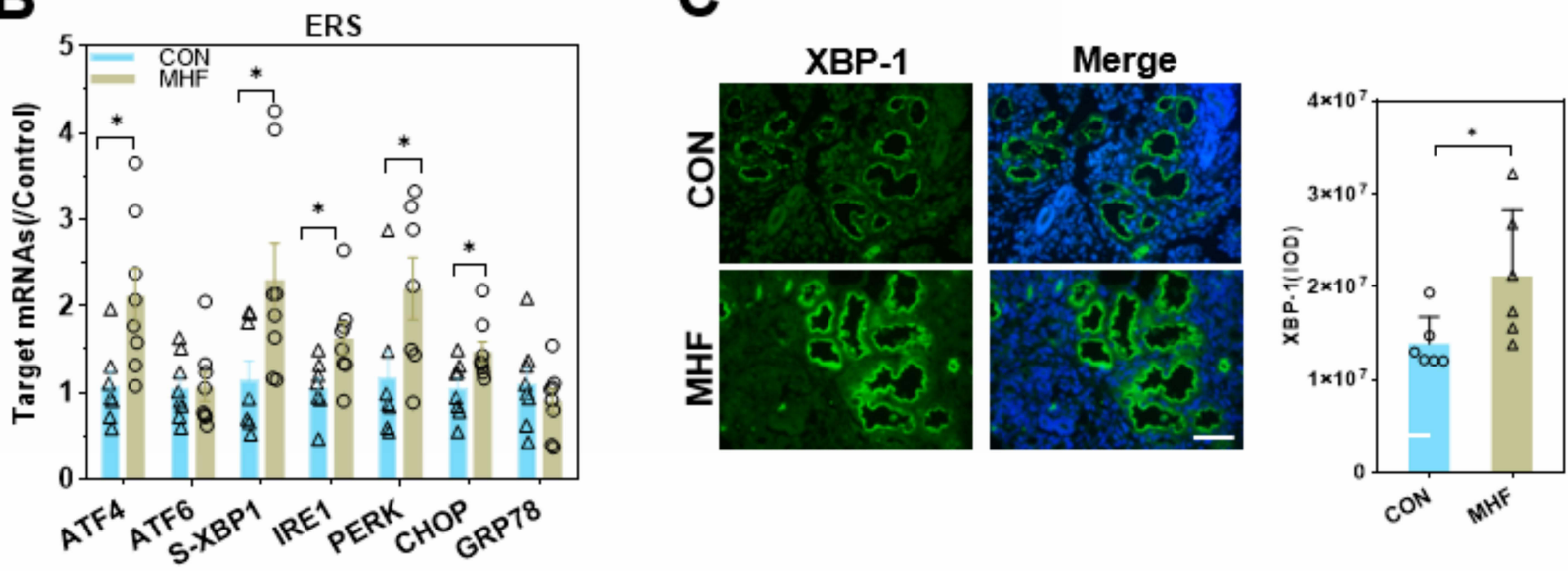

D
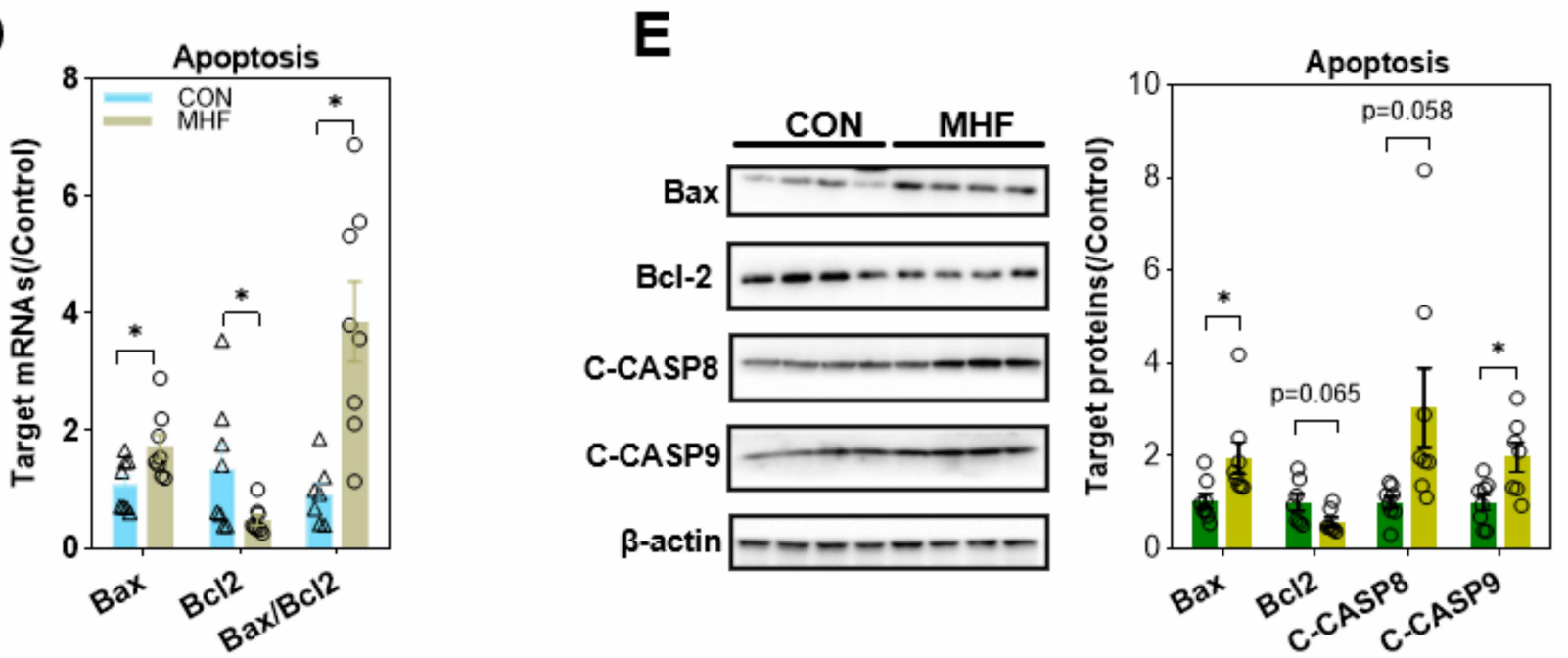

Figure 8 Effects of MHF on endoplasmic reticulum stress (ERS) and apoptosis regulators in the fetal kidney. (A) Protein expression levels of ERS regulators in the fetal kidney. ( $n=7-8$ per group, ${ }^{*} p<0.05$ vs control). (B) The qPCR analysis of the mRNA levels of ERS regulators (C) IF staining showing the expression and location of XBP-I (400x, scale bar $=50 \mu \mathrm{m}, \mathrm{n}=6$ per group, ${ }^{*} p<0.05$ vs control). (D) The qPCR analysis of the mRNA levels of Bax and BCL-2 in the fetal kidney. (E) Protein expression of apoptosis regulators in the fetal kidney. ( $n=7-8$ per group, ${ }^{*} p<0.05$ vs control).

treatment led to diminished mRNA level of ER stress regulators (PERK and CHOP) and pro-apoptotic factor Bax, while s-XBP1 was unaffected (Figure 10B and C). Western blot analysis further confirmed that PQQ restored the protein expression of CAT and Hsd17b4, decreased the protein level of TXNIP (Figure 10D). 

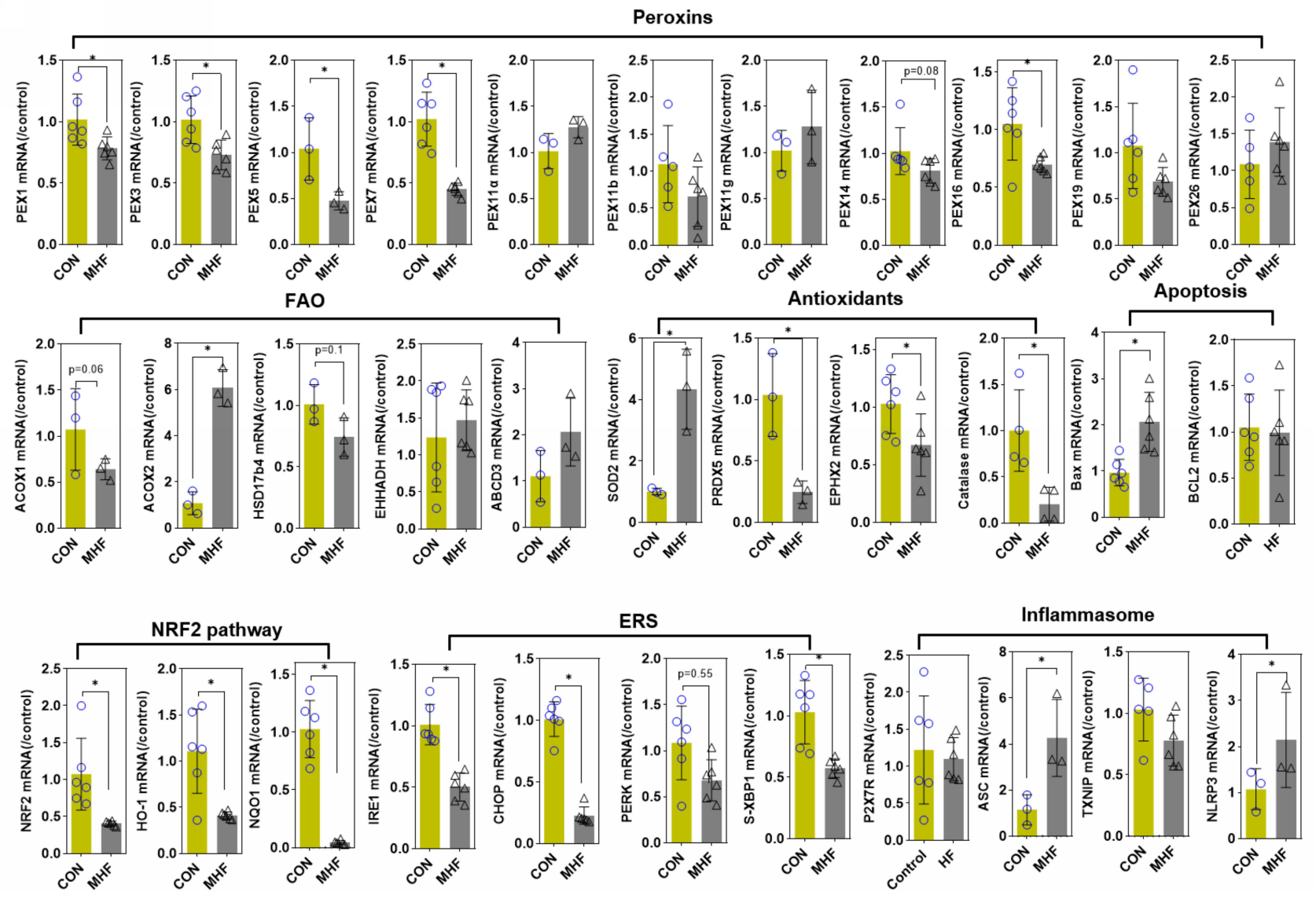

Figure 9 Effect of MHF on TECs in fetus. The qPCR analysis of the mRNA levels of PEXs/antioxidants/inflammasome in the fetal kidney. ( $n=3-6$ per group, *p $<0.05$ vs control).

\section{Discussion}

Accumulating evidence suggests that maternal obesity creates a lifelong metabolic signature on the fetal liver, kidney, and hypothalamus, which together predispose the offspring to CKD and metabolic syndrome. ${ }^{22-24}$ Rodent studies have revealed that offspring of obese mothers have enhanced OS, inflammation, and renal fibrotic injury, which last into adulthood. ${ }^{25,26}$ In the present study, consumption of a high fat diet by pregnant rats led to downregulation of the tubular epithelial marker in fetus and higher SBP in adult offspring. Our result also showed a mild decrease in birth weight of the neonates. Maternal obesity is commonly associated with fetal overgrowth. However, there are paradoxical conclusions regarding the effect of MHF on the body weight of fetus. Some reports showed that MHF had little effect on the body weight of fetus. ${ }^{27,28}$ Discrepancies in offspring development phenotype may be related to differences in the composition of high fat diets, the species, and the length of time on high fat-diet prior to mating. It cannot be rule out the possibility that the severe oxidative stress caused by excessive fatty acid affects the development of the placenta and fetal body. The important findings emerge from this research is that MHF lead to a decrease in number and dysfunction of peroxisomes, induction of ERS and apoptosis, and activation of the NLRP3 inflammasome and pyroptosis in the fetal kidney (Figure 11). And PQQ is an antioxidant that partly protects renal programming of MHF offspring from lipotoxic effects by decreasing ROS levels and suppressing activation of inflammasomes.

Peroxisomes play critical roles in multiple metabolic pathways, including FAO, polyamine oxidation, amino acid catabolism, and the oxidative part of the pentose phosphate pathway. PEX3 and 19, both involved in PMP sorting, were downregulated in the MHF fetus. Knockdown of PEX3 leads to a reduced number of peroxisomes. ${ }^{29}$ Consistent with the downregulation of PEX3, peroxisome marker PEX14, a component of the receptor docking site, was decreased in the MHF fetus. PEX5/7, matrix protein receptor, and PEX1,6 and 26, 

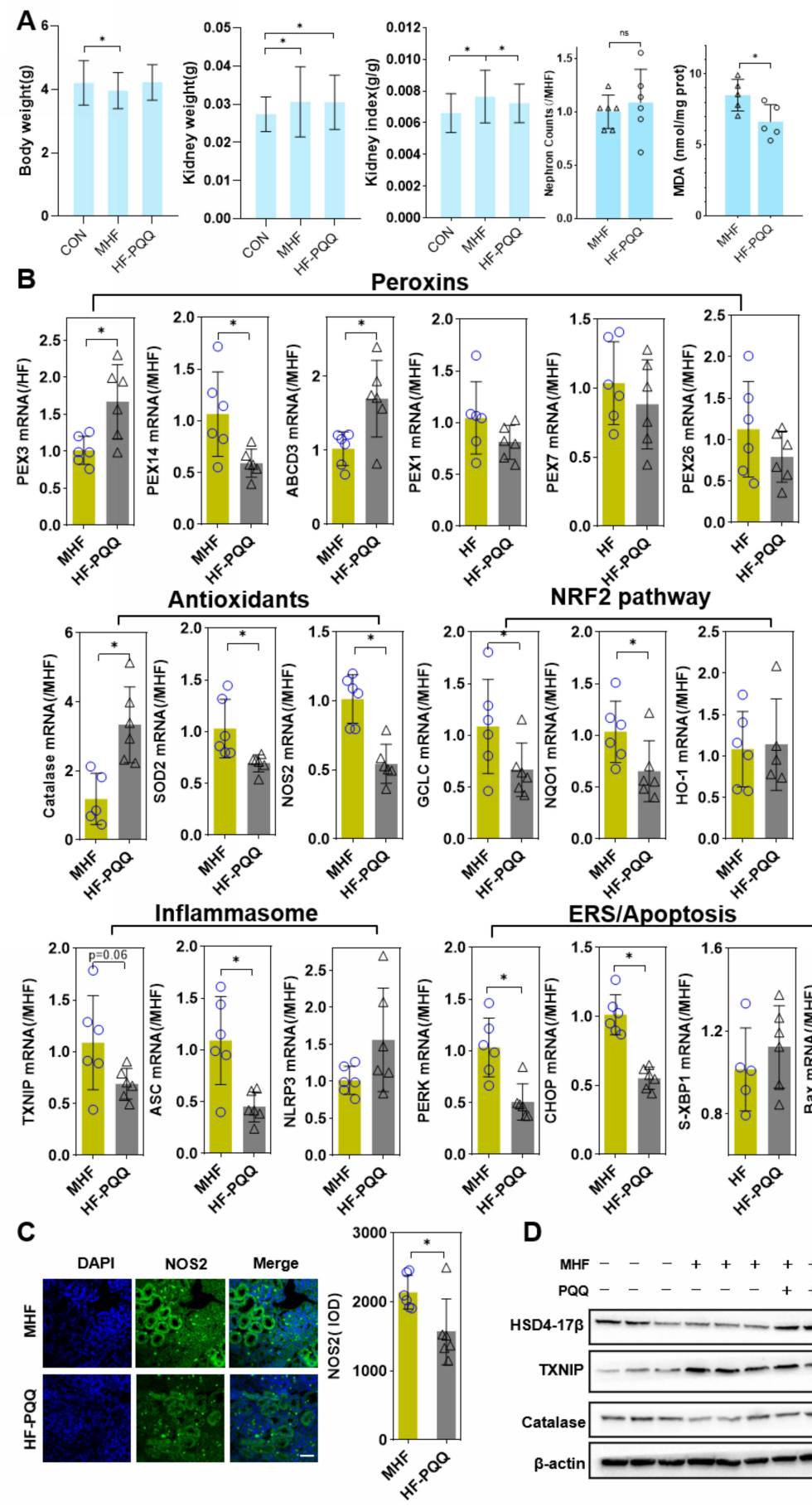

ERS/Apoptosis
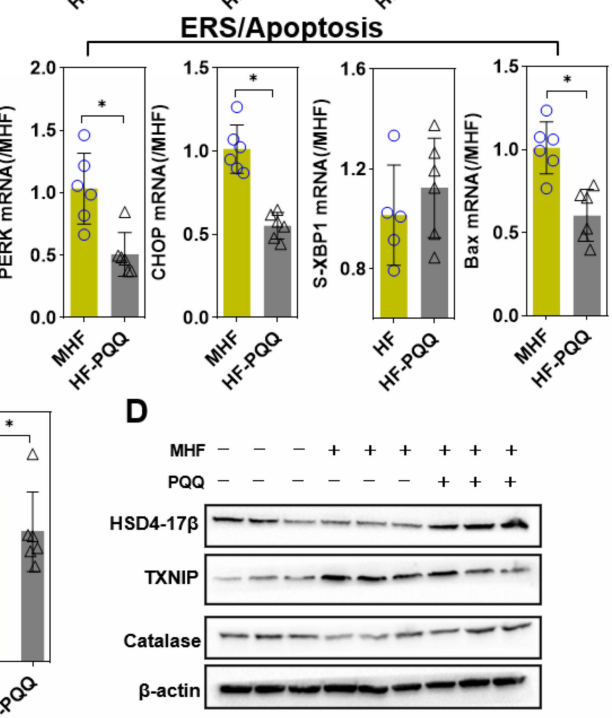

Figure 10 Effect of PQQ on renal programming. (A) Body weight, kidney weight, kidney index, and MDA level in the fetus after PQQ treatment. (B) The qPCR analysis of the mRNA level of PEXs, antioxidants, NRF2 pathway and inflammasome genes in the fetus after PQQ treatment. ( $\mathrm{n}=6$ per group, $* p<0.05$ vs MHF). (C) IF staining showing the expression of NOS2 in the fetal kidney of MHF and HF-PQQ group ( $400 \times$, scale bar $=50 \mu m, n=6$ per group, $* p<0.05$ vs MHF). (D) Western blot analysis of the expression of HsdI7b4, Txnip, and catalase in fetal kidney.

involved in receptor recycling, ${ }^{30}$ were all upregulated. These findings are valuable for the further study of impact of excess fatty acids on peroxisome biogenesis in renal tubular cells. PMP70 is an integral membrane protein involved in the transport of fatty acids across the peroxisomal membrane, which is essential for the peroxisomal oxidation of lauric and palmitic acids. The opposite tendency in the expression levels of HSD17b4 and EHHADH, which both catalyze the second step of FAO, appeared to be a compensatory effect. These results 


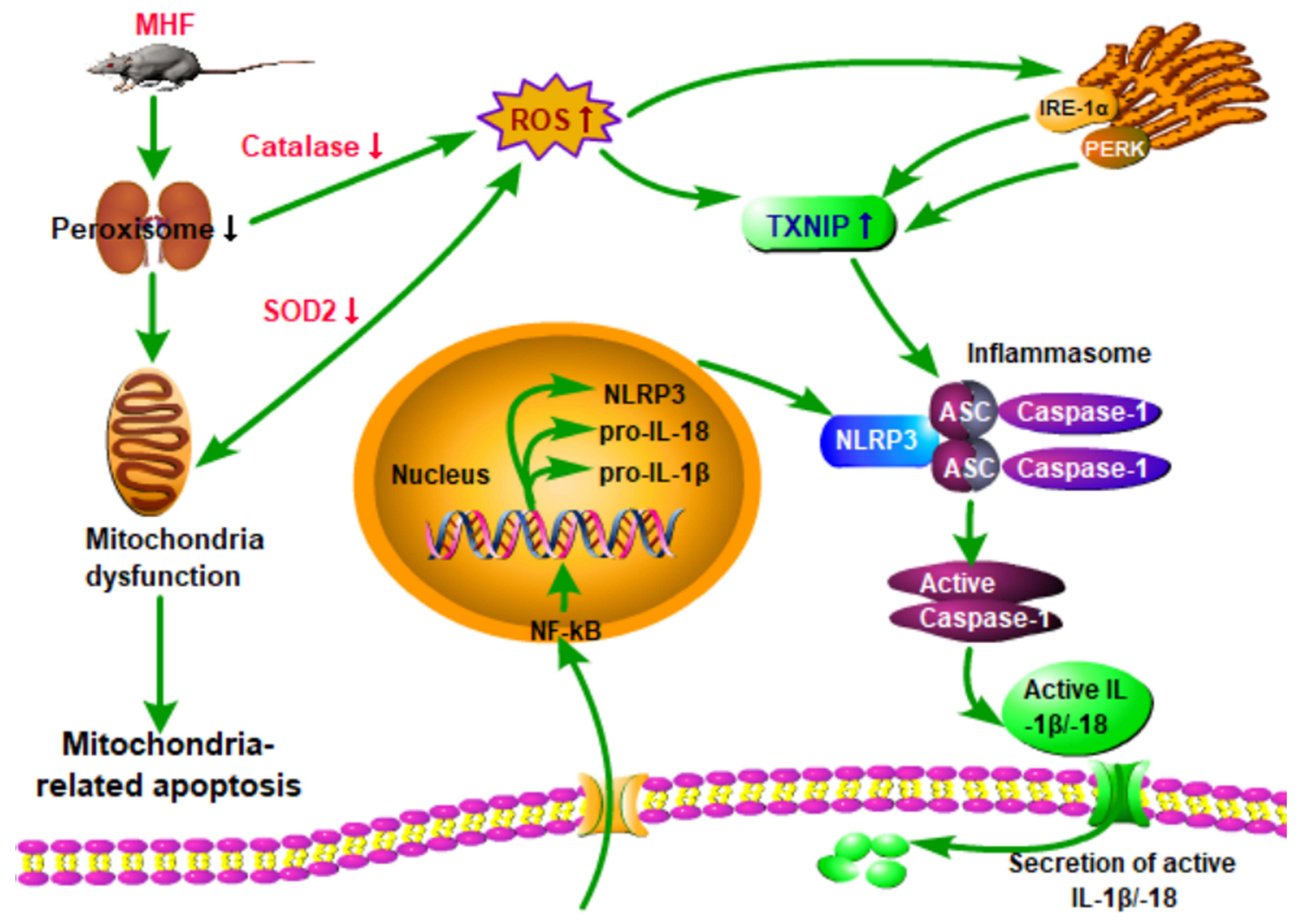

Figure II Hypothesis schematic. A unifying hypothesis depicting the key factors and cellular mechanisms influencing renal programming in response to maternal obesity.

suggested that consumption of a high fat diet by pregnant rats reduced the number of peroxisomes in the offspring. However, further analysis is needed to determine the effects on lipid profiles.

Peroxisomes in mammalian cells contain various antioxidants, including CAT, glutathione S-transferase kappa protein, and EPHX2, among others. Consumption of a high fat diet by pregnant rats led to varied expression of antioxidants in fetal kidney. The expression of EPHX2, a cytosolic and peroxisome epoxide hydrolase, ${ }^{31}$ was sharply increased in the MHF fetus. SOD2, an antioxidant located in the mitochondrial matrix, facilitates the rapid dismutation of superoxide to $\mathrm{H}_{2} \mathrm{O}_{2}$, which is then dismuted to water by CAT. MHF downregulated SOD2 and CAT in the fetal kidney, which compromises the ability of the cell to neutralize superoxide. ${ }^{32}$ NOS2 catalyzes the production of nitric oxide (NO). NO or its derivatives RNS inhibit mitochondrial respiration and induce mitochondrial permeability transition. ${ }^{33} \mathrm{~A}$ previous report indicated that peroxisomes controlled mitochondrial dynamics and the mitochondrion-dependent apoptosis pathway. Deletion of PEX3 results in mitochondrial fragmentation in mouse embryonic fibroblasts and enhances cytosolic cytochrome $\mathrm{C}$ levels and caspase activity. ${ }^{34}$ In this study, the expression levels of Bax and cleaved caspase-9 were enhanced in the MHF fetus. Collectively, these finding suggested that maternal obesity downregulated the expression of PEX3 and dysregulated ROS scavenging, resulting in mitochondrion-dependent apoptosis.

NRF2, a major antioxidant transcription factor against OS, is released from the Keap1-NRF2 complex and translocates into the cell nucleus under OS. NQO1 and HO-1 are two vital downstream antioxidant enzymes. Elevated expression of NQO1 and HO-1 in response to OS plays 
a critical role in reducing ROS levels. In this study, MHF diminished the expression of KEAP1 and augmented the expression and translocation of NRF2, as well as the associated target genes NQO1 and HO-1. Unexpectedly, although the NRF2 signaling pathway was activated, OS and inflammation were not relieved, as evidenced by the increase in MDA levels and expression of the proinflammation genes IL1 $\beta$ and IL6 in the fetal kidney. We speculated that the decreased number of peroxisomes downregulated the expression levels of SOD2 and CAT, which contributed to increased ROS content.

Notably, upregulation of NRF2 failed to inhibit activation of the NLRP3 inflammasome in the MHF fetus. The NLRP3 inflammasome is a multiprotein complex composed of a NLRP3 inflammasome sensor, ASC, and procaspase-1, which activates caspase-1 and subsequently induces the processing and secretion of the proinflammatory cytokines IL-1 $\beta$ and IL-18. Previous reports revealed the existence of the ROS/ERS/TXNIP/NLRP3 inflammasome axis in various cells. ${ }^{35,36}$ In response to ROS accumulation, ERS induces the production of TXNIP through the PERK and IRE1 $\alpha$ pathways, which binds to NLRP3 to activate the inflammasome. ${ }^{35,37,38}$ Furthermore, enhanced ROS may directly induce TXNIP to dissociate from the TRX-TXNIP complex and then bind to and activate NLRP $3 .{ }^{39}$ On the other hand, ligation of the $\mathrm{P} 2 \mathrm{X} 7$ receptor (P2X7R) by extracellular adenosine triphosphate activates the NLRP3 inflammasome, leading to secretion of proinflammatory cytokines by monocytes and macrophages. ${ }^{40}$ Based on the findings of this study, maternal obesity likely contributed to upregulation of the unfolded protein response pathway regulators IRE1 and PERK, which induced TXNIP production and subsequently activated the NLRP3 inflammasome, thus contributing to enhanced secretion of proinflammatory cytokines, eventually leading to renal fibrosis and cell injury (Figure 10). However, further studies are needed to investigate this mechanism in renal tubular epithelial cells.

AQP1 reduction suggests the dysplasia of the proximal tubules in MHF fetus. We isolated renal tubular epithelial cells from fetus and investigated the genetic changes of peroxisome/ROS/inflammasome axis. The changes in TECs are not all the same as in the renal tissue. The mRNA expression levels of most of the PEXs, PRDX5 and HSD17B4 (all located in peroxisome) were reduced in TECs. By contrast, mRNA levels of PEX1, 5, and 7 were all increased in the MHF tissues. MHF-induced upregulation of inflammasome gene NLRP3, ASC and apoptosis regulator Bax in TECs, as in tissues. However, mRNA levels of NRF2 and ERS pathway regulators were reduced, contrary to the variation tendency in tissues. The mRNA expression of endogenous antioxidants EPHX2 and SDO2 also showed opposite trends in tissues and cells. These results confirmed that MHF leads to reduction of PEXs, induces oxidative stress and inflammasome in TECs. However, contradictory results between TECs and tissues may be due to different genetic differences in various cell types in kidney caused by MHF, and need to be investigated further. It cannot be ruled out that the possibility that the primary culture TECs have undergone genetic changes after leaving the in vivo environment. If possible, it may be better to use multicolor fluorescence system to simultaneously detect both cell-specific markers and target genes in vivo.

PQQ, a redox-active o-quinone existing in plant, bacterial, and mammalian cells, is gaining increasing attention. Reportedly, PQQ exposure improves the antioxidant potential of human cells. ${ }^{41}$ However, little is known about the effect of PQQ on fetal development during pregnancy. Jonscher et $\mathrm{al}^{42}$ reported that administration of PQQ at the time of conception prevented developmental programming of hepatic lipotoxicity and inflammation. Friedman et $\mathrm{al}^{43}$ reported that treatment with PQQ prior to weaning prevented developmental programming of microbial dysbiosis and macrophage polarization, and attenuated liver fibrosis in the offspring of obese mice. Our results showed that supplementation with PQQ during pregnancy and lactation inhibited lipotoxicity-induced OS in the fetal kidney by restoring the expression of PEX3 and the CAT, thereby decreasing MDA levels. The further decrease in SOD2 levels may be due to feedback inhibition caused by ROS reduction. PQQ alleviated OS and partly inhibited MHFinduced activation of NRF2 pathway, ERS and inflammasome. Reportedly, antioxidant treatment reversed the loss of peroxisomes in Phen-treated cells. ${ }^{44}$ ROS can activate autophagy. We speculated that PQQ reduced ROS, inhibited pexophagy, then reversed peroxisome deficit. Further research into the pathways responsible for the effect of PQQ on peroxisome and inflammasomes is warranted.

There were several limitations of our study. Firstly, only the fetal kidney was investigated for the molecular changes. Further research to investigate the molecular changes in adulthood is necessary for exploring the renal programming of maternal high fat. In addition, although 
we analyzed the genetic alteration of peroxisome/ROS/ NLRP3 axis in TECs, the function and viability of the cells are not addressed, so the effect of MHF is not fully determined. Although we revealed PQQ effect on the expression of PEXs and inflammasomes, the complex cascade-like mechanism remains to be investigated.

\section{Conclusion}

Taken together, the results of the present study unveiled a previously unrecognized role of peroxisomes in renal development. The effect of PEXs gene dysregulation on ROS metabolism and the NLRP3 inflammasome may contribute to pathogenesis of CKD related to maternal nutritional status. The dietary antioxidant PQQ served a protective role against lipotoxicity during renal development and, thus, is a potential candidate to prevent renal fibrosis induced by maternal obesity.

\section{Abbreviations}

ACOX, acyl-CoA oxidase; CAT, catalase; FAO, fatty acid beta-oxidation; HSD17B4, peroxisomal 17 $\beta$ hydroxysteroid dehydrogenase type $4 ; \mathrm{H}_{2} \mathrm{O}_{2}$, hydrogen peroxide; IF, immunofluorescence; ERS, endoplasmic reticulum stress; UPR, unfolded protein reaction; NOS2, nitric oxide synthase 2; PEX, peroxin; PMP, peroxisomal membrane protein; ROS, reactive oxygen species; NLRP, NLR family, pyrin domain-containing; SOD, superoxide dismutase; TXNIP, TRX-interacting protein, ASC, Apoptosis-associated speck-like protein containing a CARD; IL, Interleukin; NRF2:NF-E2related factor 2; KEAP1, Kelch-like ECH associated protein; HO-1, heme oxygenase-1; GCLC, glutamate cysteine ligase catalytic subunit; NQO-1, NAD(P)H quinone oxidoreductase 1; CKD, chronic kidney disease; PMP, Peroxisomal membrane protein.

\section{Acknowledgments}

We would like to thank International Science Editing (http://www.internationalscienceediting.com) for editing this manuscript.

\section{Funding}

This work was supported by grant from the National Natural Science Foundation of China (No. 81971400), Outstanding Scientific Fund of Shengjing Hospital (No.201707), Natural Fund Guidance Program Project of Liaoning Province (2019-ZD-0754), Higher Education
Innovative Talent Support Plan of Liaoning Province (LR2019074).

\section{Disclosure}

The authors report no conflicts of interest in this work.

\section{References}

1. Bray GA, Kim KK, Wilding JPH. Obesity: a chronic relapsing progressive disease process. A position statement of the World Obesity Federation. Obes Rev. 2017;18(7):715-723. doi:10.1111/ obr. 12551

2. Martínez-Hortelano JA, Berlanga-Macías C, Pozuelo-Carrascosa DP, Sanabria-Martínez G, Poyatos-León R, Martínez-Vizcaíno V. Interpregnancy weight change and perinatal outcomes: a protocol for a systematic review and meta-analysis. Medicine. 2019;98(20): e15470-e15470. doi:10.1097/MD.0000000000015470

3. Chang E, Hafner H, Varghese $M$, et al. Programming effects of maternal and gestational obesity on offspring metabolism and metabolic inflammation. Sci Rep. 2019;9(1):16027. doi:10.1038/s41598019-52583-x

4. Gillman MW, Rifas-Shiman S, Berkey CS, Field AE, Colditz GA. Maternal gestational diabetes, birth weight, and adolescent obesity. Pediatrics. 2003;111(3):e221-226. doi:10.1542/peds.111.3.e221

5. Boney CM, Verma A, Tucker R, Vohr BR. Metabolic syndrome in childhood: association with birth weight, maternal obesity, and gestational diabetes mellitus. Pediatrics. 2005;115(3):e290-e296. doi:10. 1542/peds.2004-1808

6. Reynolds RM, Osmond C, Phillips DI, Godfrey KM. Maternal BMI, parity, and pregnancy weight gain: influences on offspring adiposity in young adulthood. J Clin Endocrinol Metab. 2010;95(12): 5365-5369. doi:10.1210/jc.2010-0697

7. Lee YQ, Collins CE, Gordon A, Rae KM, Pringle KG. The relationship between maternal obesity and diabetes during pregnancy on offspring kidney structure and function in humans: a systematic review. J Dev Orig Health Dis. 2019;10(4):406-419. doi:10.1017/ S2040174418000867

8. Glastras SJ, Chen H, Teh R, et al. Mouse models of diabetes, obesity and related kidney disease. PLoS One. 2016;11(8):8. doi:10.1371/ journal.pone.0162131

9. Kett MM, Denton KM. Renal programming: cause for concern? Am J Physiol Regul Integr Comp Physiol. 2011;300(4):R791-803. doi:10.1152/ajpregu.00791.2010

10. Bruce KD, Cagampang FR, Argenton M, et al. Maternal high-fat feeding primes steatohepatitis in adult mice offspring, involving mitochondrial dysfunction and altered lipogenesis gene expression. Hepatology. 2009;50(6):1796-1808. doi:10.1002/hep. 23205

11. Zambrano E, Ibáñez C, Martínez-Samayoa PM, Lomas-Soria C, Durand-Carbajal M, Rodríguez-González GL. Maternal obesity: lifelong metabolic outcomes for offspring from poor developmental trajectories during the perinatal period. Arch Med Res. 2016;47 (1):1-12. doi:10.1016/j.arcmed.2016.01.004

12. Marseglia L, Manti S, D'Angelo G, et al. Oxidative stress in obesity: a critical component in human diseases. Int J Mol Sci. 2014;16 (1):378-400. doi:10.3390/ijms16010378

13. Moldovan L, Moldovan NI. Oxygen free radicals and redox biology of organelles. Histochem Cell Biol. 2004;122(4):395-412.

14. Ermak G, Davies KJA. Calcium and oxidative stress: from cell signaling to cell death. Mol Immunol. 2002;38(10):713-721. doi:10.1016/S0161-5890(01)00108-0

15. Islinger M, Voelkl A, Fahimi HD, Schrader M. The peroxisome: an update on mysteries 2.0. Histochem Cell Biol. 2018;150(5):443-471. 
16. Zheng R, Tao L, Jian H, et al. NLRP3 inflammasome activation and lung fibrosis caused by airborne fine particulate matter. Ecotoxicol Environ Saf. 2018;163:612-619. doi:10.1016/j.ecoenv.2018.07.076

17. Seervi M, Rani A, Sharma AK, Santhosh Kumar TR. ROS mediated ER stress induces Bax-Bak dependent and independent apoptosis in response to Thioridazine. Biomed Pharmacother. 2018;106:200-209. doi:10.1016/j.biopha.2018.06.123

18. Gao W, Zhang KZ. RBP4 induces pyroptosis in cardiomyocytes via activating NLRP3/Caspase-1/GSDMD pathway in acute myocardial infarction. Eur Heart J. 2020;41(Supplement_2):ehaa946-ehaa3641. doi:10.1093/ehjci/ehaa946.3641

19. Sasakura H, Moribe H, Nakano M, Ikemoto K, Takeuchi K, Mori I. Lifespan extension by peroxidase and dual oxidase-mediated ROS signaling through pyrroloquinoline quinone in C. elegans. $J$ Cell Sci. 2017;130(15):2631-2643.

20. Akagawa M, Nakano M, Ikemoto K. Recent progress in studies on the health benefits of pyrroloquinoline quinone. Bioscience, Biotechnology, and Biochemistry. 2016;80(1):3-22. doi:10.1080/ 09168451.2015.1062715

21. Park S, Jang A, Bouret SG. Maternal obesity-induced endoplasmic reticulum stress causes metabolic alterations and abnormal hypothalamic development in the offspring. PLoS Biol. 2020;18(3): e3000296-e3000296. doi:10.1371/journal.pbio.3000296

22. Nüsken E, Turnwald E-M, Fink G, et al. Maternal high fat diet and in-utero metformin exposure significantly impact upon the fetal renal proteome of male mice. J Clin Med. 2019;8(5):663. doi:10.3390/ jcm8050663

23. Hammoud R, Pannia E, Kubant R, et al. Maternal choline intake programs hypothalamic energy regulation and later-life phenotype of male Wistar rat offspring. Mol Nutr Food Res. 2020;64(9):1901178. doi:10.1002/mnfr.201901178

24. Glastras SJ, Chen H, Tsang M, et al. The renal consequences of maternal obesity in offspring are overwhelmed by postnatal high fat diet. PLoS One. 2017;12(2):e0172644-e0172644. doi:10.1371/journal.pone. 0172644

25. Glastras SJ, Tsang M, Teh R, et al. Maternal obesity promotes diabetic nephropathy in rodent offspring. Sci Rep. 2016;6(1):27769. doi:10.1038/srep27769

26. Glastras SJ, Chen H, Pollock CA, Saad S. Maternal obesity increases the risk of metabolic disease and impacts renal health in offspring. Biosci Rep. 2018;38(2):2. doi:10.1042/BSR20180050

27. Hokke S, Puelles VG, Armitage JA, Fong K, Bertram JF, CullenMcEwen LA. Maternal fat feeding augments offspring nephron endowment in mice. PLoS One. 2016;11(8):e0161578. doi:10.1371/ journal.pone.0161578

28. Holemans K, Caluwaerts S, Poston L, van Assche FA. Diet-induced obesity in the rat: a model for gestational diabetes mellitus. Am J Obstet Gynecol. 2004;190(3):858-865. doi:10.1016/j.ajog.2003.09.025

29. Yamashita S-I, Fujiki Y. Assessing pexophagy in mammalian cells. In: Schrader M, editor. Peroxisomes: Methods and Protocols. New York: Springer New York; 2017:243-248.

30. Jansen RLM, Santana-Molina C, van den Noort M, Devos DP, van der Klei IJ. Comparative genomics of peroxisome biogenesis proteins: making sense of the PEX proteins. Front Cell Dev Biol. 2021;9:1229. doi:10.3389/fcell.2021.654163
31. Summerer S, Hanano A, Utsumi S, Arand M, Schuber F, Blée E. Stereochemical features of the hydrolysis of 9,10-epoxystearic acid catalysed by plant and mammalian epoxide hydrolases. Biochem J. 2002;366(Pt 2):471-480. doi:10.1042/bj20011778

32. Jiang W, Geng H, Lv X, et al. Idebenone protects against atherosclerosis in apolipoprotein E-deficient mice via activation of the SIRT3-SOD2-mtROS pathway. Cardiovasc Drugs Ther. 2020. doi:10.1007/s10557-020-07018-5

33. Brown GC, Borutaite V. Inhibition of mitochondrial respiratory complex I by nitric oxide, peroxynitrite and S-nitrosothiols. Biochim Biophys Acta Bioener. 2004;1658(1):44-49. doi:10.1016/j. bbabio.2004.03.016

34. Tanaka H, Okazaki T. Peroxisomes control mitochondrial dynamics and the mitochondrion-dependent apoptosis pathway. J Cell Sci. 2019;132:11.

35. Ke B, Shen W, Fang X, Wu Q. The NLPR3 inflammasome and obesity-related kidney disease. J Cell Mol Med. 2018;22(1):16-24. doi: $10.1111 /$ jcmm. 13333

36. Bryant CE, Fitzgerald KA. Molecular mechanisms involved in inflammasome activation. Trends Cell Biol. 2009;19(9):455-464. doi:10.1016/j.tcb.2009.06.002

37. Chen X, Guo X, Ge Q, Zhao Y, Mu H, Zhang J. ER stress activates the NLRP3 inflammasome: a novel mechanism of atherosclerosis. Oxid Med Cell Longev. 2019;2019:3462530. doi:10.1155/2019/3462530

38. Oslowski CM, Hara T, O'Sullivan-Murphy B, et al. Thioredoxininteracting protein mediates ER stress-induced $\beta$ cell death through initiation of the inflammasome. Cell Metab. 2012;16(2):265-273. doi:10.1016/j.cmet.2012.07.005

39. Zhou J, Chng WJ. Roles of thioredoxin binding protein (TXNIP) in oxidative stress, apoptosis and cancer. Mitochondrion. 2013;13 (3):163-169. doi:10.1016/j.mito.2012.06.004

40. Hafner-Bratkovič I, Pelegrín P. Ion homeostasis and ion channels in NLRP3 inflammasome activation and regulation. Curr Opin Immunol. 2018;52:8-17. doi:10.1016/j.coi.2018.03.010

41. Harris CB, Chowanadisai W, Mishchuk DO, Satre MA, Slupsky CM, Rucker RB. Dietary pyrroloquinoline quinone (PQQ) alters indicators of inflammation and mitochondrial-related metabolism in human subjects. J Nutr Biochem. 2013;24(12):2076-2084. doi:10.1016/j.jnutbio.2013.07.008

42. Jonscher KR, Stewart MS, Alfonso-Garcia A, et al. Early PQQ supplementation has persistent long-term protective effects on developmental programming of hepatic lipotoxicity and inflammation in obese mice. FASEB J. 2017;31(4):1434-1448. doi:10.1096/fj.201600906R

43. Friedman JE, Dobrinskikh E, Alfonso-Garcia A, et al. Pyrroloquinoline quinone prevents developmental programming of microbial dysbiosis and macrophage polarization to attenuate liver fibrosis in offspring of obese mice. Hepatol Commun. 2018;2 (3):313-328. doi:10.1002/hep4.1139

44. Jo DS, Bae D-J, Park SJ, et al. Pexophagy is induced by increasing peroxisomal reactive oxygen species in 1'10-phenanthroline-treated cells. Biochem Biophys Res Commun. 2015;467(2):354-360. doi:10.1016/j.bbrc.2015.09.153 read real quotes from published authors. mechanisms; pharmacology and novel anti-inflammatory drugs; clinical conditions involving inflammation. The manuscript managemen system is completely online and includes a very quick and fair peerreview system. Visit http://www.dovepress.com/testimonials.php to
The Journal of Inflammation Research is an international, peer-
reviewed open-access journal that welcomes laboratory and clinical findings on the molecular basis, cell biology and pharmacology of inflammation including original research, reviews, symposium reports, hypothesis formation and commentaries on: acute/chronic inflammation; mediators of inflammation; cellular processes; molecular
Dovepress

Submit your manuscript here: https://www.dovepress.com/journal-of-inflammation-research-journal 